\title{
MEASUREMENT OF CARDIAC OUTPUT USING A RADIOISOTOPE AND A SCINTILLATION COUNTER
}

\author{
BY \\ W. A. SELDON, J. B. HICKIE, AND E. P. GEORGE \\ From the Cardiovascular Unit and the Physics Department, St. Vincent's Hospital, Sydney, Australia \\ Received October 8, 1958
}

In 1897 Stewart suggested that the arterial dilution curve of a substance injected into a peripheral vein could be used to measure cardiac output. He published a number of papers on the subject but the method did not receive widespread acceptance until the classical studies of Kinsman et al. (1929) who made possible its practical application in man, when they injected a blue dye T 1824 (Evans Blue) and measured its concentration in serial samples taken from a peripheral artery by means of arterial puncture. Subsequent investigators have sought different techniques that might simplify the procedure.

The first use of a radioactive isotope as the marker substance was recorded by Nylin (1945), but in his observations the concentration curve was plotted from the counts of radioactivity in the blood following injection of radioactive iodinated serum albumin. His method utilized arterial puncture but instead of serial sampling the blood was drawn continuously through a rubber tube in contact with a scintillation counter. The wave of radioactivity passing the counter was graphed on a moving chart recorder.

In 1954 Veall et al. reported the first successful measurements of cardiac output by the use of a radio-isotope and an external scintillation counter situated over the great vessels of the chest. Shortly afterwards Huff et al. (1955) and Pritchard et al. (1955) published similar results.

The technique of Veall et al. has been used at St. Vincent's Hospital to estimate the cardiac output in twelve patients and the results are compared with figures obtained for the same patients by the Fick method. In nine instances the two determinations were made simultaneously and in three they were done under similar conditions on different days.

\section{METHOD}

The patient is given 2 grammes of potassium iodide daily, one day before and for three days after the test in order to minimize radio-iodine uptake by the thyroid gland. Although iodinated serum albumin is theoretically a stable compound, there is in fact a gradual breakdown of the molecule with liberation of free iodide ions which in this instance are radioactive. These will be taken up by the thyroid unless it is previously blocked and kept blocked. For the same reason the dose administered is kept to a minimum and it has been found by experience that 25 microcuries is a satisfactory amount.

The patient lies on a couch and a collimated scintillation counter is placed over the second left intercostal space close to the sternal border. The exact distance from the skin is not important but it is usually about one inch. It is essential only that the position of the counter relative to the patient be not altered until the end of the procedure. The counter is connected to a rate-meter, the output of which is graphed on a strip chart recorder.

Twenty-five microcuries of radioactive iodinated serum albumin is diluted up to $1 \mathrm{ml}$. with isotonic saline and injected rapidly into a suitable vein in the arm. The resulting wave of radioactivity passing through the great vessels in the chest is shown on the chart. A typical curve is 
reproduced in Fig. 1. The chart recorder is left running for ten minutes so that a record is obtained of the stable level of radioactivity in the great vessels, when the isotope is completely mixed in the total blood volume. At the end of this time $10 \mathrm{ml}$. of blood is taken into an oxalated tube. The radioactivity of this sample is compared with that of an aliquot of the injected material, so that the patient's blood volume can be determined by simple proportion. From the radioactive count per $\mathrm{ml}$. of blood and the steady ten minute reading on the chart recorder, the chart can be calibrated vertically as concentration of isotope per litre of blood. The horizontal reading on the chart represents time in seconds.

The descending limb of the curve (Fig. 1) represents the expected fall-off of concentration of the isotope interrupted at some point by the arrival of radio-active human serum albumen (RIHSA)

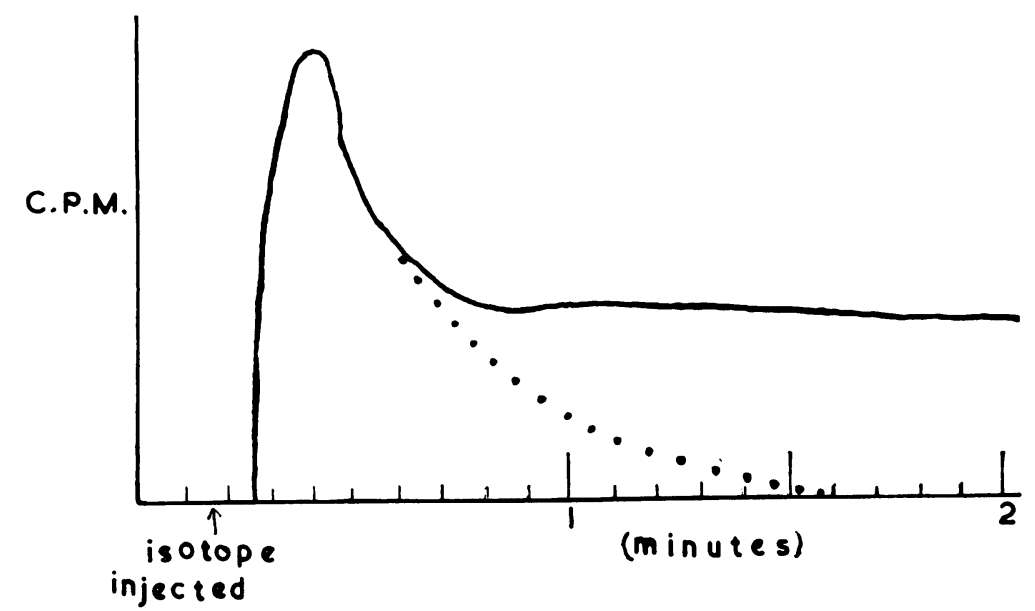

Fig. 1.- Isotope dilution curve and dotted line indicating the semi-logarithmic extrapolation of the first part of the descending limb. $\mathrm{CPM}=$ Counts per minute.

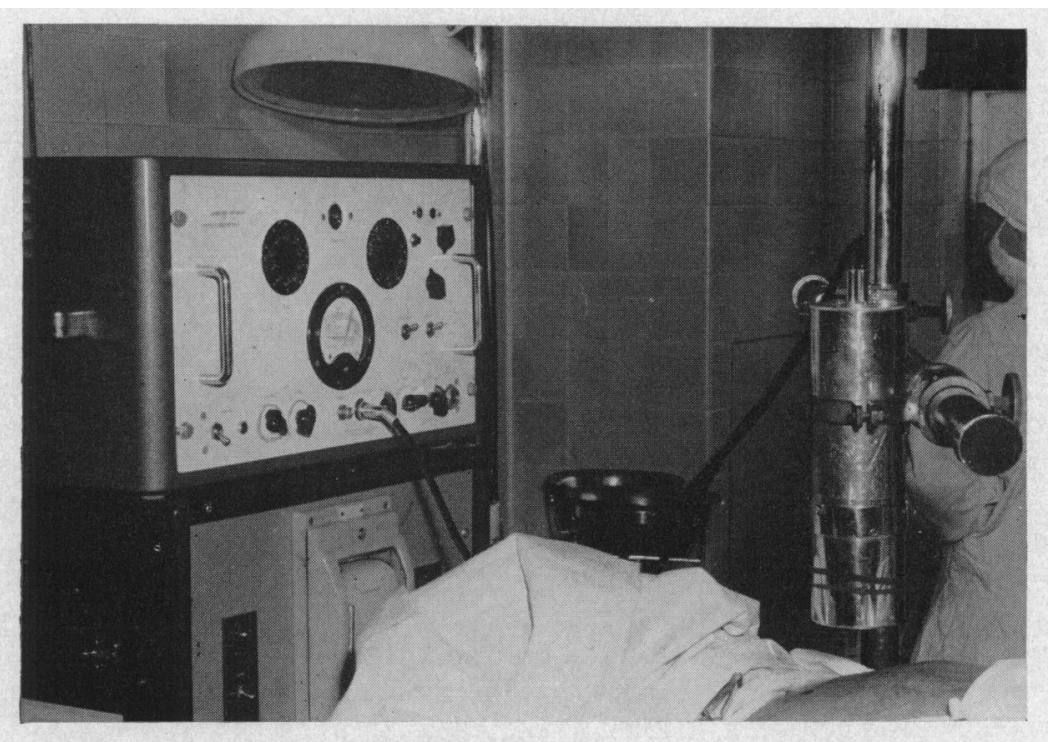

FIG. 2.-General view of the apparatus, showing scintillation counter head over the patient's chest, and on the left the rate-meter with the chart recorder underneath. (Reproduced by permission of the editor, Australasian Annals of Medicine.) 
which has completed one circulation and returned to the heart. From the work of Kinsman et al. (1929) it is known that the marker concentration decreases in an exponential fashion if recirculation does not occur. The first portion of the descending limb is therefore extrapolated semilogarithmically as shown by the dotted line in Fig. 1. The area under this "ideal" curve is then estimated graphically.

The cardiac output is derived by the application of the formula:

$$
\mathrm{CO}=\frac{\mathrm{BV} \times \mathrm{C}_{2}}{\text { Area }}
$$

where $\mathrm{CO}$ is the cardiac output in litres per minute, BV the blood volume in litres, $\mathrm{C}_{2}$ the final concentration of the isotope in the blood in microcuries per litre, and Area is the area under the curve expressed as microcuries per litre $\times$ minutes. The formula is derived as follows. Let $\mathrm{C}_{1}$ be the average concentration level during the first passage of the marker, and $T$ the mean time occupied by the first passage. Then the volume in which the marker is concentrated during the first passage will be $\mathrm{CO} \times \mathrm{T}$, and the total amount of marker, I, will be given by

but also

$$
\mathrm{I}=\mathrm{C}_{1} \times \mathrm{CO} \times \mathrm{T} \quad \cdot \quad \cdot \quad \cdot \quad \cdot \quad . \quad . \quad . \quad . \quad . \quad . \quad 2
$$

$$
\mathrm{I}=\mathrm{BV} \times \mathrm{C}_{2} \quad \text {. } \quad . \quad . \quad . \quad . \quad . \quad . \quad . \quad . \quad 3
$$

$\mathrm{C}_{1} \times \mathrm{T}$ is the area under the concentration time curve for first passage, and thus equation 2 may also be written:

$$
\mathrm{I}=\text { Area } \times \mathrm{CO}
$$

Equation 1 then follows by equating 3 and 4 .

In the Fick estimations, expired air was collected in a Douglas bag, the volume was measured by a wet gas meter and air analysis performed with a modified Haldane Grollman apparatus. Blood gas analysis was done with a Haldane apparatus.

\section{RESULTS}

Table 1 shows the results obtained in this series of estimations of the cardiac output by the Fick and radio-isotope techniques.

TABLE I

Comparison of Cardiac OUtPut Results by Fick and Isotope Techniques

\begin{tabular}{c|c|c|c}
\hline Patient & $\begin{array}{c}\text { *RIHSA output } \\
\text { litres/min. }\end{array}$ & $\begin{array}{c}\text { Fick output } \\
\text { litres/min. }\end{array}$ & $\mathrm{R} / \mathrm{F}=\overline{\mathbf{n}}$ \\
\hline $1 \times$ & $3 \cdot 7$ & $4 \cdot 1$ & 0.903 \\
$2 \times$ & $6 \cdot 1$ & $6 \cdot 8$ & 0.898 \\
$3 \times$ & 6.4 & $8 \cdot 3$ & $0 \cdot 773$ \\
4 & $6 \cdot 0$ & $5 \cdot 4$ & $1 \cdot 110$ \\
5 & $3 \cdot 7$ & $4 \cdot 5$ & $0 \cdot 820$ \\
6 & $4 \cdot 5$ & $4 \cdot 2$ & 1.070 \\
7 & $5 \cdot 8$ & $5 \cdot 5$ & 1.052 \\
8 & $4 \cdot 7$ & $4 \cdot 6$ & 1.021 \\
9 & $5 \cdot 2$ & $4 \cdot 4$ & $1 \cdot 180$ \\
10 & $4 \cdot 4$ & $4 \cdot 0$ & $1 \cdot 100$ \\
11 & $4 \cdot 5$ & $5 \cdot 3$ & $0 \cdot 850$ \\
12 & 4.4 & $4 \cdot 5$ & 0.978 \\
\hline
\end{tabular}

* Radio active human serum albumin.

$x$ Indicates non-simultaneous tests.

Mean value $\bar{n}=0.980$

Standard deviation of the mean (S.D.) $=13$ per cent

The close approach of the value $\bar{n}$ to unity indicates that there is no systematic error in the isotope method. 
The greatest difference between the two results occurred in Case 3, and it seemed that the isotope output was more consistent with the patient's clinical state than the Fick result. The latter figure gave a cardiac index of 4.9 litres per square meter per minute. This is unduly high for an apparently composed young man with a pulse rate of 86 a minute and a normal basal metabolic rate during the procedure. It is likely that the Fick method is in error in this instance. Exclusion of this case from the series would have reduced the standard deviation of the difference between the results of the two methods to 10 per cent.

\section{Discussion}

The standard deviation of 13 per cent for the ratios between the two methods is of the same order as that of Werko et al. (1949) and Etsten and Li (1954) and better than that of Hamilton et al. (1948). These groups compared the Fick method with the dye dilution method using serial arterial samples and found standard deviations of $14 \cdot 5,12 \cdot 7$, and 21 per cent respectively for the differences between the two techniques.

The standard deviation of the ratios represents the combined deviations of both the Fick and radio-isotope methods. The S.D. of the Fick technique has been assessed at 7 per cent by Seely et al. (1950) in comparison with rotameter estimations and at 10 per cent by Neely et al. (1954). In a careful study of errors in the direct Fick technique Warren et al. (1945) found that duplicate samples of expired air analysed for oxygen content varied by as much as 20 per cent in some instances with an average variation of about 10 per cent. This is presumably the main factor influencing the standard deviation of the Fick results. No figures are available for the reliability of the Fick method as carried out in our laboratory but assuming that the S.D. is as small as the best results reported, namely 7 per cent, the S.D. of the radioisotope method would be 11 per cent. If the S.D. of the Fick results are taken as 10 per cent, that of the isotope technique would be 8 per cent. These results are obtained by application of the formula.

where

$$
\sigma_{\mathrm{x}}=\sqrt{\sigma_{\mathrm{F}}^{2}+\sigma_{\mathrm{R}}^{2}}
$$

$\sigma_{\mathrm{x}}=$ S.D. of the ratios, $\overline{\mathrm{n}}$.

$\sigma_{\mathrm{F}}=$ S.D. of the Fick method.

and

$\sigma_{\mathrm{R}}=$ S.D. of the isotope method.

To sum up, the standard deviation of cardiac output determinations by the isotope method described is comparable with that of the Fick procedure.

An ideal technique for the estimation of cardiac output should fulfil the following conditions: (i) it should be simple and suitable for use in various situations such as a ward, an operating theatre, and under anæsthesia, as well as in a laboratory; (ii) it must be harmless to the patient; (iii) it should be reliable with as few sources of experimental error as possible, and (iv) it should be possible to repeat the estimation an unlimited number of times.

The Fick method has been used for many years in laboratories and in cardiac investigation units of hospitals but it fails to satisfy a number of the criteria set out above. In the first place it involves cardiac catheterization, arterial puncture, and the collection of expired air, procedures that are all time consuming and subject to technical difficulties. Furthermore if one needs to know the output, for example, during a surgical operation, the catheterization must be done initially in an $\mathrm{X}$-ray room and the patient then transferred to the operating theatre. Likewise it is impossible to employ the method in a patient who is too ill to be moved from the ward. And lastly the various procedures mentioned are hardly likely to leave the patient in a basal state, no matter how expertly performed.

The Fick technique fulfills the second condition but only partially satisfies the third. Admittedly under laboratory conditions the results are said to have a standard deviation of 7 per cent (Seely et al. 1950) but there are three different pieces of apparatus in use which may develop technical faults, the gas-meter, the air analysis apparatus, and the blood-gas analyser. Even more important is the fact 
that the Fick technique depends upon the assumption that the patient is in a steady state and it is apparent that the cardiac output is not stable in a number of patients during catheterization studies. This source of error becomes even more important if the output is determined during a surgical manœuvre such as left ventricular puncture (Brock et al. 1956; Hickie and Seldon, 1958). Lastly the number of times that the Fick estimation can be repeated depends upon the veins available for catheterization and these may be few indeed.

On the other hand the isotope method described is inherently simple requiring no more of the patient than venepuncture. It can be performed with ease in any situation. Only two pieces of apparatus are required, a rate-meter and a chart recorder, both of which are reliable and easy to maintain. Above all it causes no physical or psychological disturbances to the patient, how ever ill he may be.

With regard to the question of risk to the patient from irradiation, the dose administered by the isotope technique is much less than that received from the fluoroscopic screening necessary in placing a catheter in the pulmonary artery. I ${ }^{131}$ emits beta and gamma rays. The mean dose from beta radiation is given by the formula:

$$
\text { Radiation dose in ræntgens }=\frac{122 \times \text { millicuries }}{\text { Body weight in kilograms }}
$$

With the 25 microcuries used in this series, the dose from beta radiation is about $0.05 \mathrm{r}$ in an adult varying a little with the weight of the patient. The dose from the gamma rays is much smaller, contributing something like an additional 0.01 to $0.02 \mathrm{r}$. This total dose of about $0.07 \mathrm{r}$ is seen to be well within the internationally recommended tolerance figure of $0.3 \mathrm{r}$ per week.

The results recorded in this series show that this isotope method is at least as reliable as the Fick technique. Veall et al. (1954) and Huff et. al. (1955) have also demonstrated in two small series of cases that the external counting isotope method compares favourably with the Fick procedure. In addition this technique gives an almost instantaneous measure of output. The estimate is made over a period of about twenty seconds as compared with four minutes for the Fick technique, so that the measurement can be made simultaneously with, for example, a recording of a pressure gradient.

Estimations of cardiac output by this method can be repeated as many times as necessary, subject only to the limiting factor of radiation dosage. From the figures given above it will be seen that estimations could be done two or three times a week in an adult without exceeding the tolerance dose, and many more estimations could be done in a single week without exceeding the radiation administered during one cardiac catheterization. When the shorter life isotope $I^{132}$ is available the radiation dose could be reduced to one twentieth of that administered now, so that there would be virtually no limit to the safe number of estimations.

It is concluded that the external isotope method of determining cardiac output has numerous advantages over the conventional Fick technique and is at least as accurate as the latter method.

\section{SUMMARY}

Simultaneous cardiac output measurements have been made by the Fick and a radio-isotope version of the Hamilton method in a series of twelve patients. The technique, which involves only external counting over the patient's chest, is described and the results tabulated. The standard deviation of the isotope method is found to be close to 10 per cent, a figure frequently quoted for the Fick method. The relative merits of the two methods are discussed.

Our thanks are due to Professor Messel for the loan of some of the equipment. We are grateful to Dr. Rothfield and Mrs. Barbara Bates for making determinations of cardiac output by the Fick method and for supplying us with the data. We would like to thank the Medical Registrars of St. Vincent's Hospital for assistance in making the measurements, and Mr. Moyle and Mrs. Diana Savage for help with the isotope equipment. We are grateful to the Commonwealth X-Ray and Radium Laboratory for the supply of the radioactive tracer material. 
ADDENDUM

Since this paper was written, a report of a much larger series has been published by Shackman (1958). His findings are essentially the same as those reported here except that he used a larger dose of isotope $-100 \mu \mathrm{c}$.

\section{REFERENCES}

Brock, R. B., Milstein, B. B., and Ross, D. N. (1956). Thorax, 11, 163.

Etsten, B. E., and Li, T. H. (1954). Ancesthesiology, 15, 217.

Hamilton, W. F., Riley, R. L., Attyah, A. M., Cournand, A., Towell, D. M., Himmelstein, A., Noble, R. P., Remington, J. W., Richards, D. W., Jr., Wheeler, N. C., and Witham, A. C. (1948). Amer. J. Physiol., 153, 309.

Hickie, J. B., and Seldon, W. A. (1958). Austral. Ann. Med., 7, 112.

Huff, R. L., Feller, D. D., Judd, D. J., and Bogardus, G. M. (1955). Circ. Res., 3, 564.

Kinsman, J. M., Moore, J. W., and Hamilton, W. F. (1929). Amer. J. Physiol., 89, 322.

MacIntyre, W. J., Pritchard, W. H., Eckstein, R. W., and Friedell, H. L. (1951). Circulation, 4, 552.

Neely, W. A., Wilson, F. C., Jr., Milnor, J. P., Hardy, J. D., and Wilson, H. (1954). Surgery, 35, 22.

Nylin, G. (1945). Amer. Heart J., 30, 1.

Pritchard, W. H., MacIntyre, W. J., and Moir, T. W. (1955). J. Lab. clin. Med., 46, 939.

Seely, R. D., Nerlich, W. E., and Gregg, D. E. (1950). Circulation, 1, 1251.

Shackman, R. (1958). Clin. Sci., 17, 317.

Stewart, G. N. (1897). J. Physiol., 22, 159 (Quoted by Dow, P. (1956). Physiol. Rev., 36, 77).

Veall, N., Pearson, J. D., Hanley, T., and Lowe, A. E. (1954). Radio-isotope Conference 1954, Vol. 1, 183.

Warren, J. V., Stead, E. A., Jr., and Brannon, E. S. (1945). Amer. J. Physiol., 145, 458.

Werko, L., Lagerlof, A., Bucht, H., Wehle, B., and Holmgren, A. (1949). Scand. J. Lab. clin. Invest., 1, 109. 